\title{
Long Transmission Range Performance Evaluation on RI-MAC protocol in Wireless Sensor Networks
}

\author{
Yueh-Tiam Yong ${ }^{1}$, C.E. Tan $^{2}$, Zen, K. ${ }^{3}$ \\ PhD Student, Fac. of Comput. Sci. \& Inf. Technol., Univ. Malaysia Sarawak, Kuching, Malaysia ${ }^{1}$ \\ AP Dr, Fac. of Comput. Sci. \& Inf. Technol., Univ. Malaysia Sarawak, Kuching, Malaysia ${ }^{2}$ \\ Deputy Dean, UG, Fac. of Comput. Sci. \& Inf. Technol., Univ. Malaysia Sarawak, Kuching, Malaysia ${ }^{3}$
}

\begin{abstract}
In this paper, we are evaluating the performance receiver-initiated MAC (RI-MAC) protocol in long transmission range application focuses on the extended transmission range from $250 \mathrm{~m}$ to $850 \mathrm{~m}$ in wireless sensor nodes(WSN) using computer simulation. The reason to extend the transmission range is to apply the WSN in wider coverage with lower node density to achieve cost effectiveness, such as in the random networks. From the results obtained, that the maximum transmission range of WSN is less than $650 \mathrm{~m}$. The significant of this paper is to highlight the limitation of the current protocol when transmission range is extended to $850 \mathrm{~m}$, hence justifying the need to enhance the existing protocol for long transmission range application in WSN.
\end{abstract}

Keywords: transmission range, limitation, lower node density, wider coverage, cost effectiveness, random networks

\section{INTRODUCTION}

The size of nodes in wireless sensor network is very small and tiny [1]. Meanwhile, it consists of data processing, communication components and sensor. Therefore, the sensor node has to develop in low-cost, low-power, multifunctional sensor nodes and communicate untethered in short distances. Fortunately, the compact characters of sensor node could be developed because of the advances in micro-electro-mechanical systems (MEMS) technology [1]. However, to apply the advances character of sensor node in rural area, power source and transmission range are the main issue to be addressed. Even through, the multi-hop communication method in sensor networks can solve the problem to consume less power than the traditional single hop communication in large wide area of communication, but the cost of node deployment will be increased since the number of deployed sensor node are increased [1].

IEEE 802.11 Wireless Local Area Network (WLAN) [2] is another wireless networking technology which is currently operating at $2.4 \mathrm{GHz}$ and $5 \mathrm{GHz}$ bands. It has been one of the most popular wireless technologies in indoor environments thanks to its high data rates, easy deployment and low cost. The maximum transmission range of IEEE 802.11 system can up to $1,555 \mathrm{~m}$ system with $1 \mathrm{MHz}$ channel bandwidth based on the result from [2] and which is much longer than the required transmission range, i.e., 1,000 m. However, it consumes the transmission power up to $1000 \mathrm{~mW}$ to achieve the transmission range. Clearly, the results bring up the message about the very high energy consumption appears in IEEE 802.11 system once high transmission range is required [2].

From the descriptions of wireless sensor network and IEEE 802.11 WLAN, optimized algorithm will be designed to achieve low energy consumption and long Copyright to IJARCCE transmission range in the future. Receiver-initiated medium access control ( RI-MAC) protocol will be chosen as one of the WSN protocol for this research because from [3], RI-MAC achieved high performance in term of energy efficiency, latency and throughput compared with other previous MAC protocol [3]. However, the problem of extension transmission range in the MAC will be generated from simulation results. So that, the designation or improvement in RI-MAC is against the surface and fundamental problems. Therefore, the section $\mathrm{E}$ of this paper shown the evaluation results of RI-MAC when extending the transmission range. Meanwhile, the more detail of RI-MAC will be described in section $C$ and the simulation assumption will be described in section $\mathrm{D}$. The evaluation results and conclusion be shown in section $\mathrm{E}$ and $F$ respectively. And last, section $G$ and section $H$ shown the future work and acknowledgement.

\section{RELATED WORK :RI-MAC}

As mentioned before, high performance of energy efficiency, latency and throughput are the characters exist in RI-MAC as compared with other previous MAC protocol [3]. RI-MAC protocol is an example of asynchronous MAC protocol that has received a lot of attention [3].In order to efficiently and effectively operate over a wide range of traffic loads, RI-MAC implements receiver-initiated data transmission. A node wake up periodically to check if there are messages intended for this node by broadcasting a beacon message. A node with pending messages to be sent to the receiving node is required to stay awake and starts its transmission immediately upon receiving of the beacon messages. The receiver then sends an ACK beacon to acknowledge the received packet, and to invite new data transmission. The sender goes into sleep period after transmitted the data and www.jarcce.com 
verified that no incoming data. RI-MAC greatly reduces the medium occupancy by a pair of nodes before they reach a common active period for data transmission. The main difference between RI-MAC and the synchronous MAC protocols is that RI-MAC does not require any synchronization, and so it saves the overhead and complexity of clock synchronization [4].

After the publication of RI-MAC protocol, many new MAC protocol was improved from RI-MAC, such as ADB (An Efficient Multihop Broadcast Protocol) [5] in 2009, RC-MAC (Receiver-Centric Medium Access Control Protocol) [6] and EE-RI-MAC (energy-efficient receiverinitiated protocol) [4] in 2011. ADB MAC protocol was published in 2009. The objective to design the ADB MAC protocol is to achieve energy-efficient, low latency, and reliable multihop broadcast over asynchronous duty cycling [5]. It was using unicast transmission of DATA packet to each neighbour when it wakes up since it is asynchronous duty-cycling and the sensor nodes were waking up at different times. Unicast transmission contain acknowledgment to help a node to accurately detect whether a whether a neighbour has been reached by the broadcast and to use retransmissions to increase the reliability of the broadcast. Meanwhile, the transmitter occupying the wireless medium while waiting for each neighbour to wake up could be occupied by choosing integration of $\mathrm{ADB}$ with a receiver-initiated MAC protocol, RI-MAC [3], in which each receiver announces its wakeup with a beacon packet. The DATA transmission will start after receiving a beacon (ACK) from transmitter intended receiver. While waiting the beacon from receiver, the wireless channel is available occupied by other nodes. This to reduce delivery latency if neighbour nodes that have already received the DATA rebroadcasting it to their neighbours. ADB can efficiently support multihop broadcast in the same system by integrating with RIMAC's unicast support. Meanwhile, ADB could also be adapted for other efficient unicast MAC protocols such as WiseMAC [7]. To minimize the number of redundant transmissions, ADB efficiently distributing information on the progress of a broadcast together with DATA transmissions so that the transmissions to a node from its neighbours could be coordinated. This information also avoid transmission attempts over poor quality of the wireless links from the neighbours to the node in $\mathrm{ADB}$ [5].

In RC-MAC [6], a receiver arrange its node's packet transmissions by reusing the ACK [6]. The ACK sent to one of its nodes and can be overheard by all of its nodes due to the broadcast nature. So that the nodes packet transmissions could be scheduled by piggybacking a scheduling message. That's mean the ID of the node that can send a packet the next time, to an ACK. The ACK contain a scheduled node ID if overhearing appear[6]. This will cause a node holds its packet if the scheduled node ID is not equal to itself. Oppositely, it starts to transmit if the scheduled node ID matches its own[6]. Therefore, only the scheduled node starts to transmit while all other siblings hold their transmissions. As a result, channel collision can be significantly reduced. RC-MAC function as "pulling" data. The special many-to-one data collection traffic pattern in WSNs can be benefited.[6]

The concept behind the design of EE-RI-MAC [4] is to combine RI-MAC and AC-MAC. As explained earlier, RIMAC is a receiver-initiated protocol that requires the sender to stay awake whenever it has data to be sent. One possible way to reduce energy consumption is to allow the sender to go to sleep after waiting for a period of time and wake up periodically to listen to beacon. The sender is in active mode during the wake up interval and the receive beacon space (RBS). Considering that the energy consumption at the receiver is extremely low because a receiver only wakes up for a very short interval and goes back to sleep mode if no data is directed to it, the focus for energy saving is still at the sender[6]. In order to further reduce the period of idle period, the concept of multiple schedules in AC-MAC was adopted to allow the sender to alternately turn its radio on and off during this period. The wakeup and sleep periods of a sender are alternating in order to reduce the period of idle listening. The receiver wakes up periodically and announces its availability by broadcasting a beacon[6]. Once the beacon is detected by the sender with data waiting to be sent to this receiver, it sends the data packets immediately. During this period, receiver will stay awake to receive the packets. If nothing to be transmitted, sender and receiver will go to sleep [6].

\section{III.SIMULATION ASSUMPTION}

We used ns- 2 version 2.29 to evaluate the performance of RI-MAC in different transmission range. The comparison was done against RI-MAC only because it has been proven that RI-MAC outperforms other synchronous and asynchronous protocols [3]. We tried to model the same comparison as [3] between different types of RIMAC coverage. The propagation model used combines the free space and two-ray ground reflection models, and omnidirectional antenna was used at each node. Table 1 shows other parameters used to define the radio in the simulation and the default parameters for RI-MAC protocols are given in Table 2 [3].

TABLE 1

SIMULATION RADIO PARAMETERS.

\begin{tabular}{|c|c|}
\hline Parameter & Value \\
\hline Bandwidth & $250 \mathrm{Kbps}$ \\
\hline SIFS & $192 \mu \mathrm{s}$ \\
\hline Slot time & $320 \mu \mathrm{s}$ \\
\hline $\begin{array}{c}\text { Size of hardware } \\
\text { preamble }\end{array}$ & $6 \mathrm{~B}$ \\
\hline Size of ACK & $5 \mathrm{~B}$ \\
\hline CCA check delay & $128 \mu \mathrm{s}$ \\
\hline
\end{tabular}

TABLE 2

SIMULATION MAC PROTOCOL PARAMETERS.

\begin{tabular}{|c|c|}
\hline Parameter & RI-MAC \\
\hline Backoff window (BW) & $0-255$ \\
\hline Retry limit & 5 \\
\hline Frame & Beacon \\
\hline Frame size & $6-9$ B \\
\hline Dwell time & Variable \\
\hline
\end{tabular}


The initial value of backoff window (BW) is 32 and the receiver may adjust its according to binary exponential backoff (BEB) method that takes values of 0, 31, 63, 127 and 255. Retransmission was allowed and the maximum allowable tries was set to five. We used the same method used in [8] to evaluate the power efficiency. It is important to note that energy consumption changes significantly in different or the same radio state [9]. The initial wakeup time of each node was randomized and the sleep interval was 1 second. The transmission range and carrier sensing range of RI-MAC was also set in different values to achieve the results of different coverage. The evaluation will be applied in random network which will be explained in the next section [3].

\section{IV.EVALUATION RESULTS}

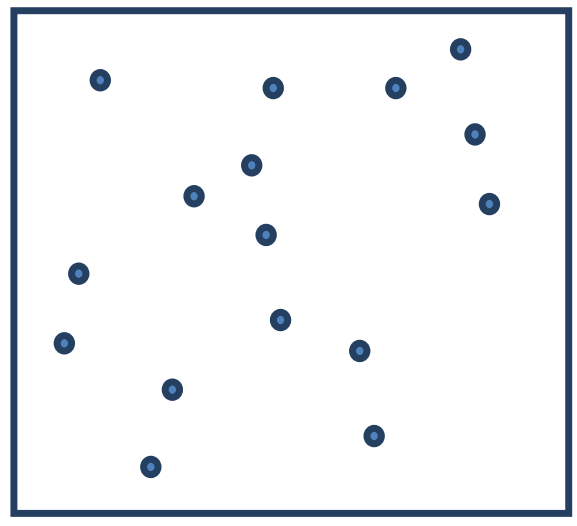

Figure 1: Random network

TABLE 3

DIFFERENT TYPES OF COVERAGE SIMULATE IN DIFFERENT SIZE OF AREA

\begin{tabular}{|c|c|}
\hline Transmission Range & Size of Area \\
\hline $250 \mathrm{~m}$ & $1000 \mathrm{~m} \times 1000 \mathrm{~m}$ \\
\hline $350 \mathrm{~m}$ & $1400 \mathrm{~m} \times 1400 \mathrm{~m}$ \\
\hline $450 \mathrm{~m}$ & $1800 \mathrm{~m} \times 1800 \mathrm{~m}$ \\
\hline $550 \mathrm{~m}$ & $2200 \mathrm{~m} \times 2200 \mathrm{~m}$ \\
\hline $650 \mathrm{~m}$ & $2600 \mathrm{~m} \times 2600 \mathrm{~m}$ \\
\hline $750 \mathrm{~m}$ & $3000 \mathrm{~m} \times 3000 \mathrm{~m}$ \\
\hline $850 \mathrm{~m}$ & $3400 \mathrm{~m} \times 3400 \mathrm{~m}$ \\
\hline
\end{tabular}

In random network (Figure 1) simulations, 100 topologies containing 50 nodes located randomly within different area was used to show the performance of RI-MAC in different transmission range. The node movement was created using node-movement generator and the RCE model with sensing range of $250 \mathrm{~m}$ until $850 \mathrm{~m}$ was used to generate one event every $60 \mathrm{~s}$ for a total of 100 events. Table 3 shows the different transmission range are simulated in different size of area. The average number of packets generated is 763 in each run.

The simulation results are presented in Figure 2, 3 and 4. Figure 2 shows cumulative distribution function (CDF) of average duty cycle. The average values for the duty cycles of all sensors for RI-MAC are dropped from $0.096 \%$ until $1.36 \%$. Clearly, the increasing of transmission range affect the higher value on the duty cycle. It means that the energy efficiency of RI-MAC drop drastically once the transmission range increase up to $650 \mathrm{~m}$.

Figure 3 gives the $\mathrm{CDF}$ of the average end-to-end delay. The average values for the delay of all sensors for RIMAC are increased from 0.09s until 800s. The difference in delay between $250 \mathrm{~m}$ and $850 \mathrm{~m}$ transmission range is about 791s. As mentioned earlier, this delay can be reduced by introducing lower coverage, which will cause the low resolution of sensor deployment.

Figure 4 gives the $\mathrm{CDF}$ of the average throughput. The average values for the throughput of all sensors for RIMAC are increased from $100 \%$ to $0 \%$ if the transmission range increase from $250 \mathrm{~m}$ until $850 \mathrm{~m}$. From the result of Figure 2 and 3, the inefficient of energy and high latency are caused by the low throughput of networks system. This is because the uncompleted data transmission affect the node always transmit the beacon to its neighbour until the number of retransmission is over. At the same time, the receivers are waiting the beacon from sender until the waiting time is over.

\section{Conclusion}

In this paper we simulated RI-MAC protocol with different transmission range and sensing range for handling dynamic traffic loads in wireless sensor networks. Generally, from the result at Figure 2, the RIMAC protocol still achieves higher energy efficiency with transmission range below $550 \mathrm{~m}$. However, the energy efficiency performance drop when the transmission range extend to $650 \mathrm{~m}$ and above. The performance of delay and throughput also change to worse once the transmission range extend to $650 \mathrm{~m}$ and above. From the results, we can conclude three limitations regarding the cause of problem from the simulation. The first limitation is the time-over of transmission and retransmission, which is related to the MAC layer. This is because the distance between nodes need take a longer time for data transmission and beacon transmission. The second limitation is because of the high frequency of retransmission, this is because of poor signal quality at certain power of time. And the last limitation, the unpredictable channel condition, mean the chances for the channel to become poor is high as compared to short transmission range.

\section{VI.FUTURE WORK}

From these three limitations, we will find out the fundamental problem which has caused the worse performance of energy efficiency, delay and throughput. Once the fundamental problem is found, new algorithm will be formulated to solve the above problems. There are two suggestions to solve the predicted problems. The first suggestions is to add-up the concept of adaptive sending of data in protocol. So that the sensor node will shift the way of transmission based on channel condition. The second suggestions is add-up a buffer at sensor node to allow delay in sending data. This will make the sensor to store the data in memory while the transmission is delaying. 


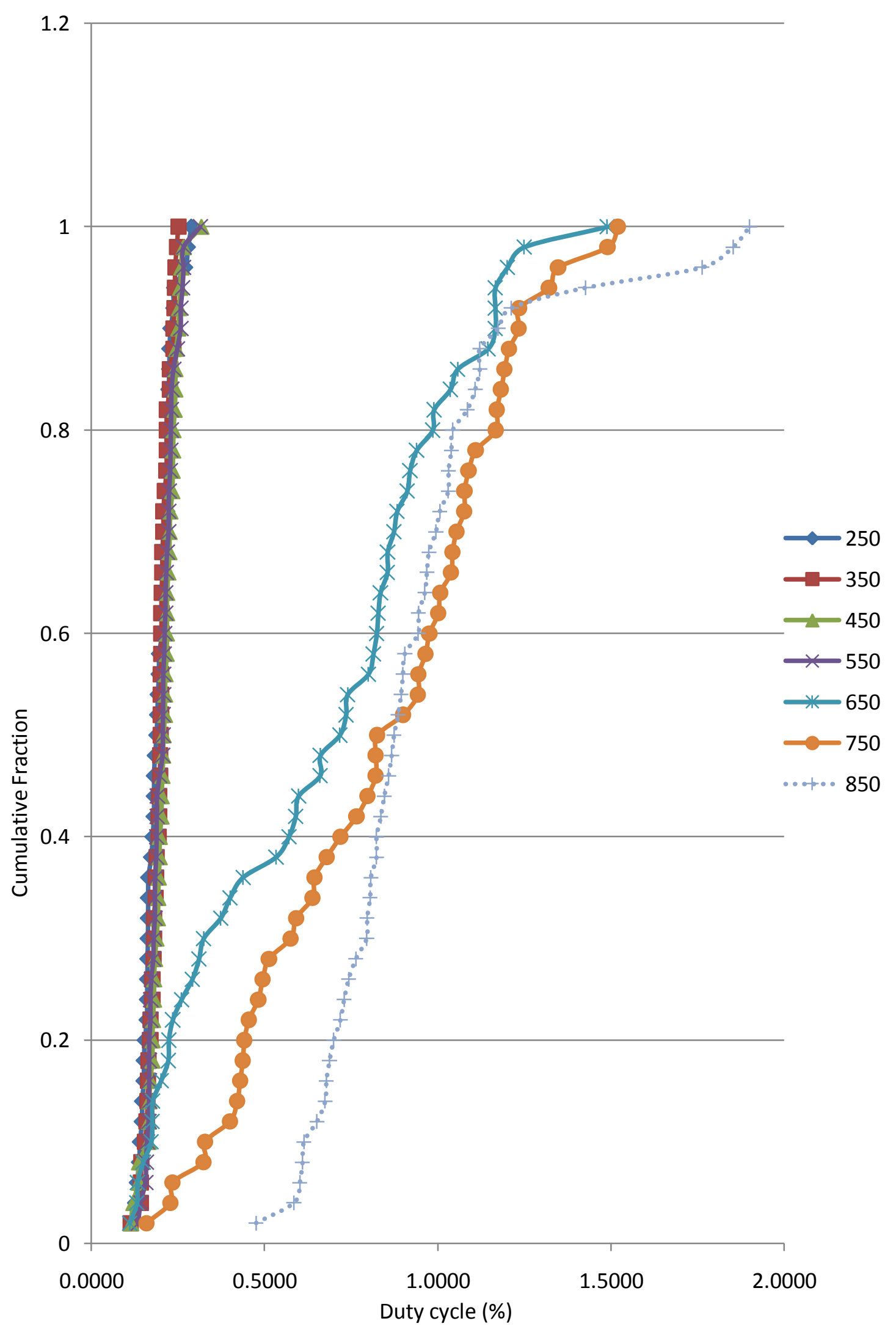

Figure 2: Average duty-cycle (\%) vs transmission Range (m) 
International Journal of Advanced Research in Computer and Communication Engineering Vol. 3, Issue 11, November 2014

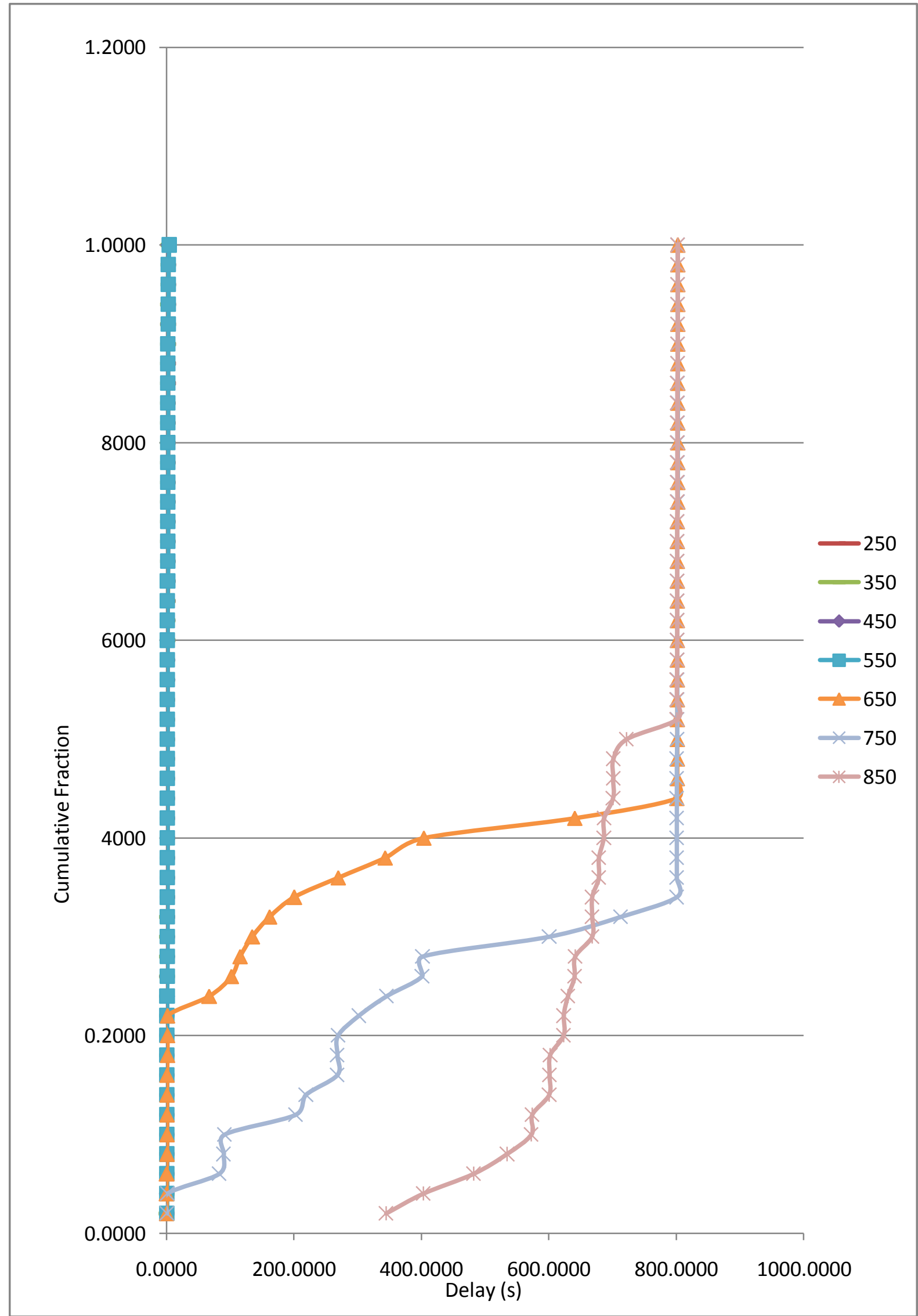

Figure 3: Delay (s) vs transmission Range (m) 
International Journal of Advanced Research in Computer and Communication Engineering Vol. 3, Issue 11, November 2014

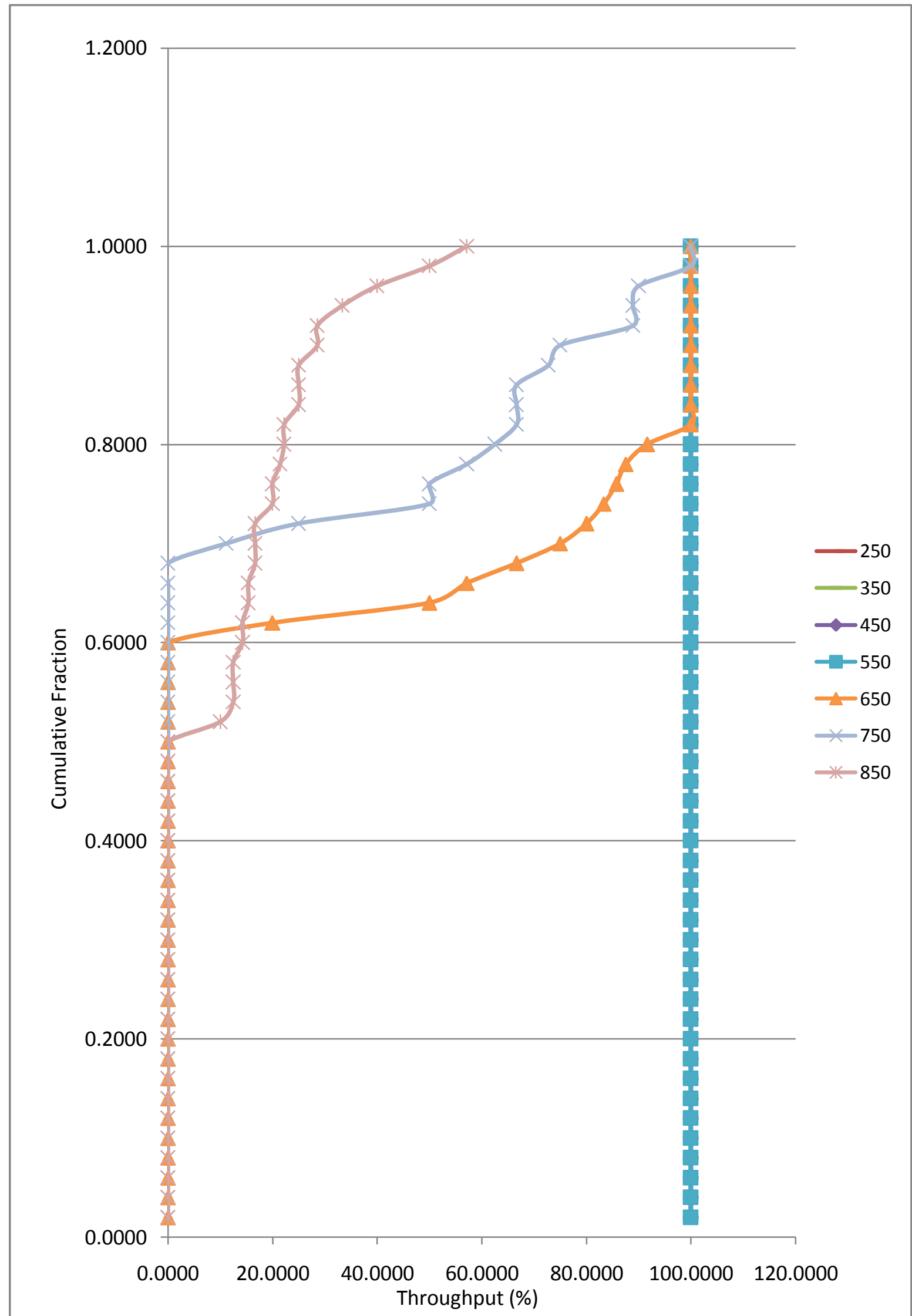

Figure 4: vs Throughput (\%) vs transmission Range (m) 


\section{ACKNOWLEDGMENT}

We thank Prof. Yanjun Sun for sharing the source code of RI-MAC and his valuable feedback. The first author also thanks the Minister of Education for supporting his studies under the Scholarship Scheme, MyBrain15.

\section{REFERENCES}

[1] I. Akyildiz, W. Su, Y. Sankarasubramaniam and E. Cayirci, 'Wireless sensor networks: a survey', Computer networks, vol. 38, no. 4, pp. 393--422, 2002.

[2] W. Sun, M. Choi and S. Choi, 'IEEE 802.11 ah: A Long Range 802.11 WLAN at Sub $1 \mathrm{GHz}^{\prime}$, Journal of ICT Standardization, vol. 1, no. 1, pp. 83--108, 2013.

[3] Y. Sun, O. Gurewitz and D. Johnson, 'RI-MAC: a receiverinitiated asynchronous duty cycle MAC protocol for dynamic traffic loads in wireless sensor networks', pp. 1--14, 2008

[4] Y. Yong, C. Chow, J. Kanesan and H. Ishii, 'EE-RI-MAC: An energy-efficient receiver-initiated asynchronous duty cycle MAC protocol for dynamic traffic loads in wireless sensor networks', International Journal of Physical Sciences, vol. 6, no. 11, pp. 2633--2643, 2011

[5] Y. Sun, O. Gurewitz, S. Du, L. Tang and D. Johnson, 'ADB: an efficient multihop broadcast protocol based on asynchronous dutycycling in wireless sensor networks', pp. 43--56, 2009.

[6] P. Huang, C. Wang, L. Xiao and H. Chen, 'RC-MAC: A receivercentric medium access control protocol for wireless sensor networks', pp. 1--9, 2010.

[7] A. El-Hoiydi and J. Decotignie, 'WiseMAC: an ultra low power MAC protocol for the downlink of infrastructure wireless sensor networks', vol. 1, pp. 244--251, 2004

[8] M. Buettner, G. Yee, E. Anderson and R. Han, 'X-MAC: a short preamble MAC protocol for duty-cycled wireless sensor networks', pp. 307--320, 2006.

[9] K. Klues, G. Hackmann, O. Chipara and C. Lu, 'A componentbased architecture for power-efficient media access control in wireless sensor networks', pp. 59--72, 2007.

\section{BIOGRAPHIES}

Yueh Tiam-Yong is an $\mathrm{PhD}$ student at the Faculty of

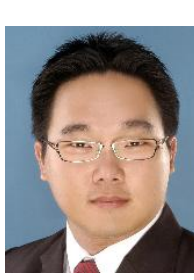
Computer Science and Information Technology, UNIMAS. He received the B.Sc. degree in Electrical Engineering from the University of Malaya, Malaysia on 2008. After the years, $\mathrm{He}$ received the MEng.Sc. degree in Wireless Communication from the University of Malaya, Malaya on 2011. From 2008 to 2011, he was at the Wireless Lab from the University of Malaya, Malaysia as Research Assistance. He is also the member of BEM (Board of Engineering Malaysia) in 2009. His major interests are Wireless Sensor Network (MAC protocol).

Chong Eng, Tan is an associate professor at the Faculty

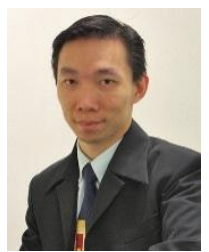
of Computer Science and Information Technology. He received the PhD degree from the University of Cambridge, UK in 2004, where he researched on throughput and performance improvement techniques applicable to OFDM based fixed broadband wireless access systems, the MEng. and BEng. degrees from Universiti Malaysia Sarawak in 1999 and 1998. He has more than 15 years experience in wireless networks and data communication related research. He is also the head of telecommunication research group in the Institute of Social Informatics and Technology Innovations (ISITI) and research fellow to the
Centre of Excellence for Image Analysis and Spatial Technologies. He is a member of the Institute of Electrical and Electronics Engineers (IEEE) and the Institution of Engineers, Malaysia (IEM). His current research interests include: Rural Long Range Wireless Networks, Green Computing Architecture, Broadband Networks, and AI for Communication Systems.

Kartinah bt Hj Zen is a Senior Lecturer of Computer Science at the Faculty of Computer Science and

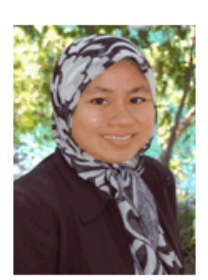
Information Technology, Universiti Malaysia Sarawak (UNIMAS). She received her Masters of Computer Science in Computer Networking (MComp Sc (Networking)) from Universiti Malaya, Malaysia. In 2006 until 2010, she pursued her Ph.D in mobile sensor network in Edith Cowan University, Australia. Her research interest is in wireless sensor networks data transmission, especially at data link and network layer. She is also a research fellow at the Centre of Excellence for Rural Informatics, and working on project in wireless sensor network. 